\title{
A STRATEGY FOR NON-STRICTLY CONVEX TRANSPORT COSTS AND THE EXAMPLE OF $\|X-Y\|^{P}$ IN $\mathbb{R}^{2 *}$
}

\author{
GUILLAUME CARLIER ${ }^{\dagger}$, LUIGI DE PASCALE , AND FILIPPO SANTAMBROGIO $^{\S}$
}

\begin{abstract}
This paper deals with the existence of optimal transport maps for some optimal transport problems with a convex but non-strictly convex cost. We give a decomposition strategy to address this issue. As a consequence of our procedure, we have to treat some transport problems, of independent interest, with a convex constraint on the displacement. To illustrate possible results obtained through this general approach, we prove existence of optimal transport maps in the case where the source measure is absolutely continuous with respect to the Lebesgue measure and the transportation cost is of the form $h(\|x-y\|)$, with $h$ strictly convex increasing and $\|\cdot\|$ an arbitrary norm in $\mathbb{R}^{2}$.
\end{abstract}

Key words. Monge-Kantorovich problem, optimal transport, existence of optimal maps, general norms.

AMS subject classifications. 49Q20, 49K30, 49J45.

\section{Introduction}

Given two Borel probability measures $\mu$ and $\nu$ on $\mathbb{R}^{d}$ and a transport cost $c: \mathbb{R}^{d} \rightarrow$ $\mathbb{R}$ the corresponding Monge problem consists in minimizing the average transport cost

$$
\int_{\mathbb{R}^{d}} c(x-T(x)) \mu(d x)
$$

among all Borel transport maps T, i.e., maps pushing forward $\mu$ to $\nu$ (which as usual is denoted by $T_{\#} \mu=\nu$ ). It is a highly nonconvex problem (whose admissible set may even be empty if $\mu$ has atoms for instance) and it is therefore relaxed to the MongeKantorovich problem that consists in minimizing

$$
\int_{\mathbb{R}^{d} \times \mathbb{R}^{d}} c(x-y) \gamma(d x, d y)
$$

over $\Pi(\mu, \nu)$, the set of Borel transport plans, i.e., of Borel probability measures on $\mathbb{R}^{d} \times \mathbb{R}^{d}$ having $\mu$ and $\nu$ as marginals. To prove existence of an optimal transport map one thus aims to prove that there is an optimal plan $\gamma$ (existence of such plans holds under very mild assumptions since the Monge-Kantorovich problem is linear) that is in fact induced by a transport map i.e., of the form $\gamma=(\mathrm{id}, T)_{\#} \mu$. To achieve this goal, one strongly uses the relations with the dual problem that consists in maximizing

$$
\int_{\mathbb{R}^{d}} \phi d \mu+\int_{\mathbb{R}^{d}} \psi d \nu
$$

subject to the constraint that $\phi(x)+\psi(y) \leq c(x-y)$. An optimal pair $(\phi, \psi)$ for the dual is called a pair of Kantorovich potentials. It is very well known (under reasonable

${ }^{*}$ Received: September 1, 2009; accepted (in revised version): January 27, 2010. Communicated by Wilfrid Gangbo.

†CEREMADE, Université Paris Dauphine, Pl. de Lattre de Tassigny, 75775, Paris Cedex 16, FRANCE (carlier@ceremade.dauphine.fr). http://www.ceremade.dauphine.fr/ carlier/

${ }^{\ddagger}$ Dipartimento di Matematica Applicata, Universitá di Pisa, Via Buonarroti 1/c, 56127 Pisa, ITALY (depascal@dm.unipi.it). http://www.dm.unipi.it/ depascal/

$\S$ CEREMADE, Université Paris Dauphine, Pl. de Lattre de Tassigny, 75775, Paris Cedex 16, FRANCE (filippo@ceremade.dauphine.fr). http://www.ceremade.dauphine.fr/ filippo/ 
assumptions on the measures) that when $c$ is strictly convex then this strategy gives an optimal transport (see [11] or section 2 below where the argument is briefly recalled; we also refer to the book [14] for a general overview and recent developments of optimal transport theory).

It is also well-known that lack of strict convexity makes the existence of an optimal transport much more delicate. Even the important case (originally considered by Monge) where $c$ is a norm was well understood only in recent years $([10,4,3,2,7,6,8])$. In the case of the Euclidean norm, for example, the direction of the displacement (we call displacements the vectors $y-x$ for $(x, y)$ in the support of an optimal $\gamma$, which becomes $T(x)-x$ in the case where this plan is induced by a transport map $T$ ) is determined by a Kantorovich potential $\phi$ : the whole space is decomposed, up to a negligible set, into a family of segments, called transport rays and parallel at each point to the gradient of $\phi$, such that $(x, y) \in \operatorname{spt} \gamma$ implies that $x$ and $y$ lay on the same segment of this family. This fixes the direction of $y-x$ but not the value of $|y-x|$. As a consequence, two important features of the problem may be underlined: on the one hand, the lack of strict convexity in the radial direction gives rise to an indeterminacy of the displacement length, but on the other hand, the problem on transport rays is one dimensional and, thus, much simpler. These observations lead to a method of proof, originally due to Sudakov [13], consisting of reduction to a one-dimensional problem on each transport ray (where the solution may be given by the monotone transport, for instance) and then gluing the solutions together. The most involved part of the proof consisted in proving that the transport rays have enough regularity so that the disintegrated measures on such rays are non-atomic (see $[4,6]$ for such a proof).

In the present paper, we consider a convex but non-strictly convex $c$. We propose to perform a decomposition that takes advantage of the fact that, whenever the displacement is not fully determined, it means that it lies in some face of $c$, but on such a face the cost is affine, and is therefore unchanged if we replace the transport plan by another one which has the same marginals and satisfies the further constraint that the displacement belongs to the face. In this spirit, our strategy can be compared to Sudakov's, yet it is different since there is no real analogue of transport rays here. Instead, our approach, detailed in section 2, involves "face restricted problems" that are optimal transport problems with convex constraints on the displacement. Such constrained problems have, we believe, their own interest and motivations (e.g., due to connections with $L^{\infty}$ transportation problems as studied in [9]) and we will address some of them in section 3 . We will avoid here subtle disintegration arguments to glue together the face restricted problems in general but will instead illustrate in section 4 how our method easily produces an optimal transport map in the case of $c(z)=h(\|z\|)$ with $h$ strictly convex increasing and $\|\cdot\|$ an arbitrary norm in $\mathbb{R}^{2}$. The contributions of this paper are then:

- a general decomposition strategy to deal with convex but non-strictly convex costs,

- a contribution to constrained transport problems,

- the proof of existence of optimal transport maps for a class of costs in $\mathbb{R}^{2}$. 


\section{Strategy of decomposition}

In this section, we outline a possible procedure to study existence of an optimal transport for the Monge-Kantorovich problem

$$
\min \left\{\int_{\mathbb{R}^{d} \times \mathbb{R}^{d}} c(x-y) \gamma(d x, d y): \gamma \in \Pi(\mu, \nu)\right\},
$$

where $c$ is convex but not strictly convex. Not all of the steps listed below can always be carried out in full generality. In the following sections, we will detail some cases where this is possible and illustrate some applications.

The decomposition is based on the following steps.

- Consider an optimal plan $\gamma$ and look at the optimality conditions by means of a solution $(\phi, \psi)$ of the dual problem. From the fact that

$$
\phi(x)+\psi(y)=c(x-y) \text { on } \operatorname{spt} \gamma \quad \text { and } \quad \phi(x)+\psi(y) \leq c(x-y)
$$

one deduces that if $x$ is a differentiability point for $\phi$ (which is denoted $x \in$ $\operatorname{Diff}(\phi))$,

$$
\nabla \phi(x) \in \partial c(x-y)
$$

which is equivalent to

$$
x-y \in \partial c^{*}(\nabla \phi(x)) .
$$

Let us define

$$
\mathcal{F}_{c}:=\left\{\partial c^{*}(p): p \in \mathbb{R}^{d}\right\},
$$

which is the set of all values of the multi-valued subdifferential map of $c^{*}$ (for the standard convex analysis notions of subdifferential and Legendre Transform, we refer to [12]). These values are those convex sets where the function $c$ is affine, and they will be called faces of $c$. Indeed, to explain why the faces of a convex function $c$ are the values of the subdifferential of its Legendre transform $c^{*}$, let us mention that $c$ is affine on a region $K$ if and only if a common vector belongs to the subdifferential of all the points of $K$. Hence, we can say $x \in F \Leftrightarrow v \in \partial f(x)$, which becomes $x \in F \Leftrightarrow x \in \partial f^{*}(v)$ (thanks to the crucial result in convex analysis $v \in \partial f(x) \Leftrightarrow x \in \partial f^{*}(v)$ ); this means $F=\partial f^{*}(v)$.

Thanks to $(2.1)$, for every fixed $x$ all the points $y$ such that $(x, y)$ belongs to the support of an optimal transport plan are such that the difference $x-y$ belong to a same face of $c$. When $c^{*}$ is differentiable (which is the same as $c$ being strictly convex), these faces are singleton and then this is the way to obtain a transport map, since only one $y$ is admitted for every $x$.

Equation (2.1) also enables one to classify the points $x$ as follows. For every $K \in \mathcal{F}_{c}$ we define the set

$$
X_{K}:=\left\{x \in \operatorname{Diff}(\phi): \partial c^{*}(\nabla \phi(x))=K\right\} .
$$

Hence $\gamma$ may be decomposed into several subplans $\gamma_{K}$ according to the criterion $x \in X_{K}$. If $K$ varies among all possible faces this decomposition covers $\gamma$-almost all pairs $(x, y)$ (if $\phi$ is differentiable $\mu$-a.e.). Moreover, if $(x, y)$ belongs to spt $\gamma$ and $x$ to $\operatorname{Diff}(\phi)$, then $x \in X_{K}$ implies $x-y \in K$. 
If the set $\mathcal{F}_{c}$ is finite or countable, we can define

$$
\gamma_{K}:=\gamma_{\mid X_{K} \times \mathbb{R}^{d}}
$$

which is the simpler case. Actually, in this case, the marginal measures $\mu_{K}$ and $\nu_{K}$ of $\gamma_{K}$ (i.e., its images under the maps $(x, y) \mapsto x$ and $(x, y) \mapsto y$, respectively) are submeasures of $\mu$ and $\nu$, respectively. In particular $\mu_{K}$ inherits absolute continuity from $\mu$. This is often useful for proving existence of transport maps.

If $\mathcal{F}_{c}$ is uncountable, in some cases one can still rely on a countable decomposition by considering the set $\mathcal{F}_{c}^{\text {multi }}:=\left\{K \in \mathcal{F}_{c}: K\right.$ is not a singleton $\}$. If $\mathcal{F}_{c}^{\text {multi }}$ is countable, then one can separate those $x$ such that $\partial c^{*}(\nabla(\phi(x))$ is a singleton (where a transport already exists) and look at a decomposition for $K \in \mathcal{F}_{c}^{\text {multi }}$ only.

In some other cases, it could be useful to bundle together different possible $K$ 's so that the decomposition is countable, even if coarser. We will give an example of this last type in section 4.

- This decomposition reduces the transport problem to a superposition of transport problems of the type

$$
\min \left\{\int_{\mathbb{R}^{d} \times \mathbb{R}^{d}} c(x-y) \gamma(d x, d y): \gamma \in \Pi\left(\mu_{K}, \nu_{K}\right), \operatorname{spt} \gamma \subset\{(x, y): x-y \in K\}\right\} .
$$

The advantage is that the cost $c$ restricted to $K$ is easier to study. For instance, if $K$ is a face of $c$, then $c$ is affine on $K$ and in this case the transport cost does not depend any more on the transport plan.

- If $K$ is a face of $c$ the problem is reduced to finding a transport map from $\mu_{K}$ to $\nu_{K}$ satisfying the constraint $x-T(x) \in K$, knowing a priori that a transport plan satisfying the same constraint exists.

In some cases (for example if $K$ is a convex compact set with non-empty interior) this problem may be reduced to an $L^{\infty}$ transport problem. In fact, if one denotes by $\|\cdot\|_{K}$ the (gauge-like) "norm" such that $K=\left\{x:\|x\|_{K} \leq 1\right\}$, one has

$$
\min \left\{\max \left\{\|x-y\|_{K},(x, y) \in \operatorname{spt} \gamma\right\}, \gamma \in \Pi(\mu, \nu)\right\} \leq 1
$$

and the question is whether the same result would be true if one restricted the admissible set to transport maps only (passing from Kantorovich to Monge, say). The answer would be positive if a solution of (2.2) were induced by a transport map $T$ (which is true if $\mu_{K} \ll \mathcal{L}^{d}$ and $K=\overline{B(0,1)}$, see [9], but is not known in general). Moreover, the answer is also positive in $\mathbb{R}$ where the monotone transport solves all the $L^{p}$ problems, and hence the $L^{\infty}$ as well.

- A positive answer may be also given if one is able to select, for instance by a secondary minimization, a particular transport plan satisfying $\operatorname{spt} \gamma \subset\{(x, y)$ : $x-y \in K\}$ which is induced by a map. This leads to the very natural question of solving

$$
\min \left\{\int_{\mathbb{R}^{d} \times \mathbb{R}^{d}}\left[\frac{1}{2}|x-y|^{2}+\chi_{K}(x-y)\right] d \gamma: \gamma \in \Pi(\mu, \nu)\right\},
$$

or, more generally, transport problems where the cost function involves convex constraints on $x-y$. These problems are studied in section 3. For instance, 
in the quadratic case above, we can say that the optimal transport $T$ exists and is given by $T(x)=x-\operatorname{proj}_{K}(\nabla \phi(x))\left(\operatorname{proj}_{K}\right.$ denoting projection on $\left.K\right)$, provided two facts hold:

- $\mu$ is absolutely continuous, so that we can assure the existence of a (possibly approximate) gradient of $\phi$ up to a $\mu$-negligible set, under mild regularity assumptions on $\phi$;

- an optimal potential $\phi$ does actually exist, in a class of functions (Lipschitz, BV) which are differentiable almost everywhere, at least in a weak sense.

- In order to apply the study of convex-constrained problems to the original problem with $c(x-y)$, the first issue (i.e., absolute continuity) does not pose any problem if the decomposition is finite or countable, while it is nontrivial in the case of disintegration, and presents the same kind of difficulties as in Sudakov's solution of Monge's problem. For interesting papers related to these kinds of problems, see for instance $[5,6]$.

- As far as the second issue is concerned, this is much more delicate, since in general there are no existence results for potentials with non-finite costs. In particular, a counterexample has been provided by Caravenna when $c(x, y)=$ $|x-y|+\chi_{K}(x-y)$ where $K=\mathbb{R}^{+} \times \mathbb{R}^{+} \subset \mathbb{R}^{2}$ and it is easily adapted to the case of quadratic costs with convex constraints. On the other hand, it is easy to think that the correct space to set the dual problem in Kantorovich theory for these kinds of costs would be $B V$ since the constraints on $x-y$ enable one to control the increments of the potentials $\phi$ and $\psi$ on some directions, thus giving some sort of monotonicity. Yet, this is not sufficient for finding a bound in $B V$ if an $L^{\infty}$ estimate is not available as well, and the counterexample that we mentioned - which gives infinite values to both $\phi$ and $\psi$ - exactly proves that these kinds of estimates are hard to prove.

REMARK 2.1. An interesting example that could be approached by decomposition as we propose here is that of crystalline norms (a problem that has been already solved by a different method in [2]). In this case, the faces of the cost $c$ are polyhedral cones but, if the support of the two measures are bounded, we can suppose that they are compact convex polyhedra. This means, thanks to the considerations above, that it is possible to perform a finite decomposition and to reduce the problem to some $L^{\infty}$ minimizations for norms whose unit balls are polyhedra. In particular, solving the $L^{\infty}$ problem for crystalline norms is enough to solve the usual $L^{1}$ optimal transport problem for the crystalline norms.

REMARK 2.2. A simple example that can be completely solved in any dimension through this strategy is the Monge problem for the cost $c(z)=(|z|-1)_{+}^{2}$, which vanishes for displacements smaller than one. In this case it is easy to see that $\mathcal{F}_{c}^{\text {multi }}$ has only one element, which is given by the Euclidean ball $\overline{B(0,1)}$. The associated $L^{\infty}$ problem has been solved in [9] and this is enough to prove the existence of an optimal transport.

\section{Constrained transport problems}

In this section we see two useful examples of transport problem under the constraint $x-y \in K$. Since the cost we use, due to this constraint, is lower semicontinuous but not finitely valued it is well-known that duality holds (the minimum of the Kantorovich problem coincides with the supremum of the dual one), but existence of 
optimizers in the dual problem is not guaranteed. As we underlined in section 2, this is a key point and what we present here will always assume that this existence holds true. In section 4 we will show a relevant example in which this is actually the case.

\subsection{Strictly convex costs with convex constraints}

We start from a general and abstract transport problem with convex constraints; in order to present these constraints we introduce the characteristic function $\chi_{K}$ of a convex set $K$ defined as

$$
\chi_{K}(x)= \begin{cases}0 & \text { if } x \in K, \\ +\infty & \text { otherwise }\end{cases}
$$

The first case we consider is the one where the cost function is strictly convex, which makes the problem easier to solve.

TheOREM 3.1. Let $\mu, \nu$ be two probability measures on $\mathbb{R}^{d}, K$ a closed and convex subset of $\mathbb{R}^{d}$ and $h: \mathbb{R}^{d} \rightarrow \mathbb{R}$ a strictly convex function. Let $c(z)=h(z)+\chi_{K}(z)$ : then the transport problem

$$
\min \left\{\int_{\mathbb{R}^{d} \times \mathbb{R}^{d}} c(x-y) \gamma(d x, d y), \gamma \in \Pi(\mu, \nu)\right\}
$$

admits a unique solution, which is induced by a transport map $T$, provided that a transport plan with finite cost exists and the dual problem admits a solution $(\phi, \psi)$ where $\phi$ is approximately differentiable $\mu$-a.e.

Proof. As usual, consider an optimal transport plan $\gamma$ and optimal potentials $\phi$ and $\psi$. The optimality conditions on optimal transport plans and optimal potentials for this problem read as

$$
\phi(x)+\psi(y)=h(x-y) \text { on } \operatorname{spt} \gamma \subset\{x-y \in K\}
$$

and

$$
\phi(x)+\psi(y) \leq h(x-y) \text { for all }(x, y): x-y \in K
$$

and, if $\phi$ is differentiable at $x$, they lead to

$$
\nabla \phi(x) \in \partial c(x-y)=\partial h(x-y)+\mathcal{N}_{K}(x-y),
$$

where $\mathcal{N}_{K}(z):=\left\{l \in \mathbb{R}^{d}: l \cdot(y-z) \leq 0, \forall y \in K\right\}$ is the normal cone to $K$ at $z$ (but the same may be easily checked in case $\phi$ is only approximately differentiable). We used the fact that the subdifferential of the sum $h$ and $\chi_{K}$ is the sum of their subdifferentials since $h$ is real-valued and hence continuous (see [12]). Equation (3.1) is satisfied by the true gradient of $\phi$ if it exists but it stays true for the approximate gradient if $\phi$ is only approximately differentiable.

Whenever a vector $l$ and a point $\bar{z} \in K$ satisfy

$$
l \in \partial h(\bar{z})+\mathcal{N}_{K}(\bar{z})
$$

thanks to the convexity of $h$ and $K$ this gives that $\bar{z}$ minimizes $K \ni z \mapsto h(z)-l \cdot z$. Since $h$ is strictly convex this gives the uniqueness of $\bar{z}$, which will depend on $l$. We will denote it by $\bar{z}(l)$. 
In this case, we get $x-y=\bar{z}(\nabla \phi(x))$, which is enough to identify $y=T(x):=$ $x-\bar{z}(\nabla \phi(x))$ and proves existence of a transport map which is necessarily unique.

REMARK 3.2. Notice that, in the case $h(z)=\frac{1}{2}|z|^{2}$ the point $\bar{z}(l)$ will be the projection of $l$ on $K$, which gives the nice formula

$$
T(x)=x-\operatorname{proj}_{K}(\nabla \phi(x)),
$$

i.e., a generalization of the usual formula for the optimal transport in the quadratic case.

REMARK 3.3. Notice also that the abstract assumption that the potential $\phi$ is differentiable $\mu$-a.e. is usually enforced by assuming that $\mu$ is absolutely continuous and proving that one can choose a potential $\phi$ which is either Lipschitz continuous or $B V$.

3.2. Strictly convex costs of one variable and convex constraints. Let $\mu \ll \mathcal{L}^{2}$ and $\nu$ be two probability measures in $\mathbb{R}^{2}$, let $K$ be a convex subset of $\mathbb{R}^{2}$ such that $\stackrel{\circ}{K} \neq \emptyset$, and consider the cost

$$
c(x-y)=h\left(x_{1}-y_{1}\right)+\chi_{K}(x-y),
$$

for an increasing and strictly convex function $h: \mathbb{R} \rightarrow \mathbb{R}$.

Theorem 3.4. Assume that there exist $\gamma \in \Pi(\mu, \nu)$ and $\phi, \psi$ Lipschitz such that

$$
\begin{gathered}
\phi(x)+\psi(y) \leq c(x-y) \quad \forall x, y \\
\phi(x)+\psi(y)=c(x-y) \quad \gamma-a . e .
\end{gathered}
$$

Then there exists an optimal transport map for the cost c between $\mu$ and $\nu$.

REMARK 3.5. The assumption above implies that $\gamma$ is an optimal plan and the pair $(\phi, \psi)$ is optimal for the dual problem. In order to have the existence of an optimal $\gamma$ it is enough to assume that there exists at least one $\sigma \in \Pi(\mu, \nu)$ such that

$$
\int_{\mathbb{R}^{2} \times \mathbb{R}^{2}} c(x-y) \sigma(d x, d y)<\infty .
$$

Again we recall that this assumption is not enough to have the existence of an optimal couple for the dual problem.

Proof. As $\mathcal{L}^{2}\left(\mathbb{R}^{2} \backslash \operatorname{Diff}(\phi)\right)=0$, then $\mu\left(\mathbb{R}^{2} \backslash \operatorname{Diff}(\phi)\right)=0$ and we can restrict our attention to the points of differentiability of $\phi$. For each $(x, y) \in \operatorname{spt}(\gamma)$ such that $x \in \operatorname{Diff}(\phi)$ by (3.2) and (3.3) we obtain

$$
\nabla \phi(x) \in h^{\prime}\left(x_{1}-y_{1}\right) e_{1}+\mathcal{N}_{K}(x-y) .
$$

By the convexity of the functions involved (3.4) is equivalent to

$$
x-y \in \arg \min _{z}\left\{h\left(z_{1}\right)-\nabla \phi(x) \cdot z+\chi_{K}(z)\right\} .
$$

Let $S$ be the set of those $x$ such that the set on the right-hand side of (3.4) is a singleton

$$
\arg \min _{z}\left\{h\left(z_{1}\right)-\nabla \phi(x) \cdot z+\chi_{K}(z)\right\}=\{p\}
$$


then, for $x \in S, y$ is uniquely determined by

$$
y=x-p .
$$

Let us consider a decomposition of $\gamma$ in two parts: $\gamma=\gamma_{\mid S \times \mathbb{R}^{2}}+\gamma_{\mid S^{c} \times \mathbb{R}^{2}}$. The first part of the decomposition is already supported on the graph of a Borel map. As far as the second part is concerned, we will prove the existence of a transport map which gives the same cost and the same marginals. This will be done by a sort of one-dimensional decomposition according to the following observations.

Whenever the set on the right-hand side of (3.4) is not a singleton, then, by the convexity of the function $h\left(z_{1}\right)-\nabla \phi(x) \cdot z$, it is a convex subset of $K$. Even more, by the strict convexity of $h$, there exists a number $m(\nabla \phi(x))$, such that

$$
\arg \min _{z}\left\{h(z)-\nabla \phi(x) \cdot z+\chi_{K}(z)\right\} \subset\left\{z \cdot e_{1}=m(\nabla \phi(x))\right\} \bigcap K .
$$

We claim that if $\arg \min _{z}\left\{h(z)-\nabla \phi(x) \cdot z+\chi_{K}(z)\right\}$ has more than one element then $x$ is a local maximum of $\phi$ on the line $x+t e_{2}$. In fact, assume that there exist two points $y=\left(m(\nabla \phi(x)), y_{2}\right)$ and $\tilde{y}=\left(m(\nabla \phi(x)), \tilde{y}_{2}\right)$ such that $x-y \in \arg \min _{z}\{h(z)-\nabla \phi(x)$. $\left.z+\chi_{K}(z)\right\}$ and $x-\tilde{y} \in \arg \min _{z}\left\{h(z)-\nabla \phi(x) \cdot z+\chi_{K}(z)\right\}$ and assume without loss of generality that $\tilde{y}_{2}<y_{2}$. As $x-y \in K$, then $x+t e_{2}-y \in K$ for small and positive $t$ (because it belongs to the segment $[x-y, x-\tilde{y}]$ ), then

$$
\begin{gathered}
\phi\left(x+t e_{2}\right)+\psi(y) \leq h\left(x_{1}-m(\nabla \phi(x))\right) \\
\phi(x)+\psi(y)=h\left(x_{1}-m(\nabla \phi(x))\right)
\end{gathered}
$$

and substracting the second equation from the first

$$
\phi\left(x+t e_{2}\right)-\phi(x) \leq 0 .
$$

The same inequality is obtained for small, negative $t$ using $\tilde{y}$.

We now introduce the set $M_{n}$ of local maxima of radius $\frac{1}{n}: M_{n}:=\left\{x: \phi\left(x+t e_{2}\right) \leq\right.$ $\left.\phi(x) \forall t \in\left[-\frac{1}{n}, \frac{1}{n}\right]\right\}$. The set $M_{n}$ is closed. Let $M_{n}^{i}:=M_{n} \cap\left\{x: x \cdot e_{2} \in\left[\frac{i}{n}, \frac{i+1}{n}\right]\right\}$. It is a general fact that, whenever a real function is restricted to the intersection $I$ of its set of local maxima of radius $r$ with a set whose diameter is no more than $r$, then this function is constant on $I$. Actually, if $a, b \in I$, the value at $a$ cannot be smaller than the value of $b$ due to the local maximality of $a$ which arrives up to $b$, and since the roles of $a$ and $b$ may be interchanged then the value is the same. In our case, this means that $\phi$ is vertically constant on $M_{n}^{i}$. Thus, there exists a function $\tilde{\phi}$ depending only on the variable $x_{1}$ such that $\tilde{\phi}$ coincide with $\phi$ on $M_{n}^{i}$. Then on the intersection of the set of the Lebesgue points of $\left(M_{n}^{i}\right)$ with $\operatorname{Diff}(\phi)$, we have $\nabla \phi=a p p \nabla \phi=a p p \nabla \tilde{\phi}$ and the latter approximate gradient depends only on $x_{1}$ which implies that $\nabla \phi$ is vertically constant on a subset of full measure of $M_{n}^{i}$. The disjoint union $S^{c}=\cup_{n, i}\left[S^{c} \cap\left(M_{n}^{i} \backslash M_{n-1}\right)\right]$ induces the decomposition

$$
\gamma_{\mid S^{c} \times \mathbb{R}^{2}}=\sum_{n, i} \gamma_{n, i}
$$

where $\gamma_{n, i}:=\gamma_{\mid\left[S^{c} \cap\left(M_{n}^{i} \backslash M_{n-1}\right)\right] \times \mathbb{R}^{2}}$.

Denote $\mu_{n, i}$ and $\nu_{n, i}$ to be the marginals of $\gamma_{n, i}$. Clearly $\mu_{n, i}$ is absolutely continuous with respect to $\mathcal{L}^{2}$. Consider the disintegration according to $x_{1}$

$$
\mu_{n, i}=a_{n, i}\left(x_{1}\right) \cdot \mathcal{L}^{1} \otimes \mu_{n, i}^{x_{1}}
$$


with $\mu_{n, i}^{x_{1}} \ll \mathcal{L}^{1}$ for almost every $x_{1}$, where $a_{n, i}\left(x_{1}\right) \cdot \mathcal{L}^{1}$ is the projection of $\mu_{n, i}$ on the first variable (which is absolutely continuous as well). Analogously, we define $\nu_{n, i}^{y_{1}}$ as the disintegration of $\nu_{n, i}$ with respect to $y_{1}$.

Let $(x, y) \in \operatorname{spt} \gamma_{n, i}$ : by definition of $m(\nabla \phi(x))$, we necessarily have $y_{1}=x_{1}-$ $m(\nabla \phi(x))$. Since $\operatorname{spt} \gamma_{n, i} \subset M_{n}^{i}$, we know that both $\phi$ and $\nabla \phi$ are vertically constant on $\operatorname{spt} \gamma_{n, i}$, which implies that $m(\nabla \phi(x))$ actually depends on $x_{1}$ only. If we denote $T_{n, i}$ to be the function associating to $x_{1}$ the number $x_{1}-m(\nabla \phi(x))$, we have $\operatorname{spt} \gamma_{n, i} \cap\left\{(x, y): x \cdot e_{1}=x_{1}\right\} \subset\left\{(x, y): y \cdot e_{1}=T_{n, i}\left(x_{1}\right)\right\}$.

Let us now disintegrate $\gamma_{n, i}$ with respect to $x_{1}$ and let us denote $\gamma_{n, i}^{x_{1}}$ to be this disintegration (that is $\gamma_{n, i}^{x_{1}}$ is a measure on variables $\left(x_{2}, y_{1}, y_{2}\right)$ and $\gamma_{n, i}=a_{n, i}\left(x_{1}\right)$. $\left.\mathcal{L}^{1} \otimes \gamma_{n, i}^{x_{1}}\right)$. It follows from what we have shown above that the marginal of $\gamma_{n, i}^{x_{1}}$ on the $y_{1}$ variable is $\delta_{T_{n, i}\left(x_{1}\right)}$. Hence, the measure $\gamma_{n, i}^{x_{1}}$, is completely determined by the other marginal, that we will call $\eta_{n, i}^{x_{1}}$, so that $\gamma_{n, i}^{x_{1}}=\delta_{T_{n, i}\left(x_{1}\right)} \otimes \eta_{n, i}^{x_{1}}$. The measures $\eta_{n, i}^{x_{1}}$ are measures on variables $\left(x_{2}, y_{2}\right)$, and we define $\theta_{n, i}^{x_{1}}$ to be the marginal of $\eta_{n, i}^{x_{1}}$ on variable $y_{2}$. By construction, we thus have:

$$
\int u\left(x_{1}\right) v\left(y_{1}\right) w\left(y_{2}\right) \gamma_{n, i}(d x, d y)=\int u\left(x_{1}\right) v\left(T_{n, i}\left(x_{1}\right)\right)\left(\int w\left(y_{2}\right) \theta_{n, i}^{x_{1}}\left(d y_{2}\right)\right) a_{n, i}\left(x_{1}\right) d x_{1}
$$

for all test-functions $u, v$ and $w$.

It is then enough to replace $\gamma_{n, i}$ with a new transport plan of the form (id $\times$ $\left.S_{n, i}\right)_{\#} \mu_{n, i}$ with the following properties:

$$
\begin{gathered}
S_{n, i}\left(x_{1}, x_{2}\right)=\left(T_{n, i}\left(x_{1}\right), S_{n, i}^{x_{1}}\left(x_{2}\right)\right), \\
\left(S_{n, i}^{x_{1}}\right)_{\#} \mu_{n, i}^{x_{1}}=\theta_{n, i}^{x_{1}}, \quad x_{2}-S_{n, i}^{x_{1}}\left(x_{2}\right) \in K_{x_{1}-T_{n, i}\left(x_{1}\right)},
\end{gathered}
$$

where we denote, for every $t$, the section of $K$ at level $t$ by $K_{t}:=\{s:(t, s) \in K\}$. The fact that (id $\left.\times S_{n, i}\right)_{\#} \mu_{n, i}$ actually is a transport plan between $\mu_{n, i}$ to $\nu_{n, i}$ easily follows from its construction and (3.5).

Since the $h$-part of the cost only depends on $x_{1}-y_{1}$ and $\gamma_{n, i}$ and the transport plan issued by the map $S_{n, i}$ realize the same displacements as far as the first coordinates are concerned, this part of the cost does not increase. Moreover, the constraint $\left(x_{1}-y_{1}, x_{2}-y_{2}\right) \in K$, which amounts to the requirement that $x_{2}-y_{2}$ belongs to a segment $K_{x_{1}-y_{1}}$, is preserved, thanks to the last condition above. Hence, the two transport plans have exactly the same cost.

We are only left to find, for every $x_{1}$, the map $S_{n, i}^{x_{1}}$. For this, it is enough to choose the monotone transport map from $\mu_{n, i}^{x_{1}}$ to $\theta_{n, i}^{x_{1}}$. This map is well defined because the first measure is absolutely continuous (in particular, there are no atoms). The constraint is preserved because $\gamma_{n, i}^{x_{1}}$ satisfied it, and the monotone transport map is optimal for any convex transport cost of $x_{2}-y_{2}$ : if one applies it to the convex function $\chi_{K_{x_{1}-y_{1}}}$, this proves that if a transport plan satisfies the constraint $x_{2}-y_{2} \in K_{x_{1}-y_{1}}$, then the same is true for the one induced by a monotone increasing map.

Notice that the whole map $S_{n, i}$ we are using is measurable since in every ambiguous case we chose the monotone transport map. 
4. Application: $c(x-y)=h(\|x-y\|)$ in $\mathbb{R}^{2}$

THEOREM 4.1. Let $\mu, \nu$ be probability measures compactly supported in $\mathbb{R}^{2}$, with $\mu \ll \mathcal{L}^{2}$, let $\|\cdot\|$ be an arbitrary norm of $\mathbb{R}^{2}$, and let $h: \mathbb{R}^{+} \rightarrow \mathbb{R}^{+}$to be a strictly convex and increasing function. Denote $c$ to be the function $c(z)=h(\|z\|)$, which is still a convex function. Then the transport problem

$$
\min \left\{\int_{\mathbb{R}^{d} \times \mathbb{R}^{d}} c(x-y) \gamma(d x, d y), \gamma \in \Pi(\mu, \nu)\right\}
$$

admits at least a solution which is induced by a transport map $T$.

Proof. The strategy to prove this theorem is almost the one described in section 2 , with the additional trick of bundling together some faces of the convex function $h(\|x-y\|)$. The boundary of the unit ball of the norm $\|\cdot\|$ has a countable number of flat parts (they are countable since each one has a positive length, otherwise it would not be a flat part, and we cannot have more than a countable quantity of disjoint positive length segments in the boundary). We call $F_{i}$, with $i=1,2, \ldots$ the closures of these flat parts and we then associate to each face $F_{i}$ the cone $K_{i}=\left\{t z, t \geq 0, z \in F_{i}\right\}$.

Consider an optimal transport plan $\gamma$ and a pair of Kantorovich potentials $(\phi, \psi)$ (these objects exist since the cost is continuous). From the general theory of optimal transport and what we underlined in section 2, $\phi$ is locally Lipschitz and if $(x, y) \in \operatorname{spt} \gamma$ and $x$ is a differentiability point of $\phi$, then $\nabla \phi(x) \in \partial c(x-y)$. This may be re-written as

$$
x-y \in \partial c^{*}(\nabla \phi(x)) .
$$

We classify the points $x$ according to $\partial c^{*}(\nabla \phi(x))$. This subdifferential may be either a singleton or a segment (it cannot be a set of dimension two because otherwise $c$ would be affine on a set with non-empty interior, which is in contradiction with the strict convexity of $h)$. We call $X_{0}$ the set of those $x$ such that $\partial c^{*}(\nabla \phi(x))$ is a singleton. If, instead, it is a segment, it is a segment of points which share a single vector in the subdifferential of $c$. This means, thanks to the shape of $c$, that it is necessarily an homothety of one face $F_{i}$ :

$$
x-y \in \partial c^{*}(\nabla \phi(x))=t(x) F_{i(x)} \subset K_{i(x)} .
$$

We denote $X_{i}$ to be the set of points $x$ where $\partial c^{*}(\nabla \phi(x))$ is an homothety of $F_{i}$.

The disjoint decomposition given by the sets $X_{i}$ induces a corresponding decomposition $\gamma=\sum_{i \geq 0} \gamma_{i}$ where $\gamma_{i}=\gamma_{\mid X_{i} \times \mathbb{R}^{2}}$. Each sub-transport plan $\gamma_{i}$ is an optimal transport plan between its marginals $\mu_{i}$ and $\nu_{i}$, which are submeasures of $\mu$ and $\nu$, respectively. In particular, each measure $\mu_{i}$ is absolutely continuous.

Consider the optimal transport problem for the cost $c$. We now build a solution induced by a transport map by modifying $\gamma$ in the following way: $\gamma_{0}$ is already induced by a transport map since for every $x$ we only have one $y$; every $\gamma_{i}$ for $i \geq 1$ will be replaced by a new transport plan with the same marginals which does not increase the cost, thanks to Theorem 3.4. Since $\gamma_{i}$ sends $\mu_{i}$ to $\nu_{i}$, is optimal for the cost $c$, and satisfies the additional condition $\operatorname{spt} \gamma_{i} \subset\left\{(x, y): x-y \in K_{i}\right\}$, it is also optimal for the cost $c(x-y)+\chi_{K_{i}}(x-y)$. The advantage of this new cost is that $c$ depends on one variable only, when restricted to $K_{i}$. Actually there exists a new basis $\left(e_{1}, e_{2}\right)$ such that $\|z\|=z_{1}$ for every $z \in K_{i}$ which is written as $\left(z_{1}, z_{2}\right)$ in this new basis (the direction of the vector $e_{1}$ being the normal direction to $F_{i}$ ). Theorem 3.4 precisely states that the optimal transport problem for a cost which is given by a strictly 
convex function of one variable plus a constraint imposing that $x-y$ belongs to a given convex set admits a solution induced by a transport map, provided the first measure is absolutely continuous and some $\gamma, \phi$ and $\psi$ satisfying equations (3.2) and (3.3) exist. This last assumption is exactly satisfied by taking $\gamma_{i}$ and the original potentials $\phi$ and $\psi$ for the problem with $\operatorname{cost} c$ from $\mu$ to $\nu$, since they satisfied

$$
\begin{array}{r}
\phi(x)+\psi(y) \leq h(\|x-y\|) \leq h(\|x-y\|)+\chi_{K_{i}}(x-y), \\
\phi(x)+\psi(y)=h(\|x-y\|)=h(\|x-y\|)+\chi_{K_{i}}(x-y) \text { on } \operatorname{spt} \gamma_{K_{i}} .
\end{array}
$$

Acknowledgments. The work of the authors has been partially supported by the University Paris Dauphine (CEREMADE) and the University of Pisa through the project "Optimal transportation and related topics". G.C. and F.S. gratefully acknowledge the support of the Agence Nationale de la Recherche through the project "ANR-07-BLAN-0235 OTARIE". The authors wish to thank the Centro de Ciencias de Benasque Pedro Pascual (CCBPP) where this work was completed during the program "Partial differential equations, optimal design and numerics", August 23 September 4, 2009.

\section{REFERENCES}

[1] L. Ambrosio, Lecture notes on optimal transportation problems, Mathematical aspects of evolving interfaces (Funchal, 2000), Lecture Notes in Math., Springer, Berlin, 1812, 1-52, 2003.

[2] L.Ambrosio, B. Kirchheim and A. Pratelli, Existence of optimal transport maps for crystalline norms, Duke Math. J., 125(2), 207-241, 2004.

[3] L. Ambrosio and A. Pratelli, Existence and stability results in the $L^{1}$ theory of optimal transportation, Optimal transportation and applications (Martina Franca, 2001), Lecture Notes in Math., Springer, Berlin, 1813, 123-160, 2003.

[4] L.A. Caffarelli, M. Feldman and R.J. McCann, Constructing optimal maps for Monge's transport problem as a limit of strictly convex costs, J. Amer. Math. Soc., 15(1), 1-26, 2002.

[5] L. Caravenna and S. Daneri, The disintegration of the Lebesgue measure on the faces of a convex function, preprint, 2009, available at http://cvgmt.sns.it

[6] L. Caravenna, A proof of Sudakov theorem with strictly convex norms, preprint, 2008, available at http://cvgmt.sns.it.

[7] T. Champion and L. De Pascale, The Monge problem for strictly convex norms in $\mathbb{R}^{d}$, J.E.M.S., to appear, preprint, available at http://cvgmt.sns.it.

[8] T. Champion and L. De Pascale, The Monge problem in $\mathbb{R}^{d}$, preprint, 2009, available at http://cvgmt.sns.it.

[9] T. Champion, L. De Pascale and P. Juutinen, The $\infty$-Wasserstein distance: local solutions and existence of optimal transport maps, SIAM J. Math. Anal., 40(1), 1-20, 2008.

[10] L.C. Evans and W. Gangbo, Differential Equations Methods for the Monge-Kantorovich Mass Transfer Problem, Mem. Amer. Math. Soc., 137, 1999.

[11] W. Gangbo and R.J. McCann, The geometry of optimal transportation, Acta Math., 177, 113$161,1996$.

[12] R.T. Rockafellar, Convex Analysis, Princeton University Press, Princeton, N. J., 1970.

[13] V.N. Sudakov, Geometric problems in the theory of infinite-dimensional probability distributions, Cover to Cover Translation of Trudy Mat. Inst. Steklov, 141, 1976. Proc. Steklov Inst. Math., 2(i-v), 1-178, 1979.

[14] C. Villani, Topics in Optimal Transportation, Graduate Studies in Mathematics, Amer. Math. Soc., 58, 2003. 